Journal of Applied Analysis

Vol. 12, No. 1 (2006), pp. 109-118

\title{
ON AN ASYMPTOTIC BOUNDARY VALUE PROBLEM FOR SECOND ORDER DIFFERENTIAL EQUATIONS
}

\author{
K. SZYMAŃSKA \\ Received April 23, 2004 and, in revised form, April 26, 2005
}

\begin{abstract}
The existence of at least one solution to a nonlinear second order differential equation in $\mathbb{R}^{k}$ on the semi-infinite with the first derivative vanishing at infinity is proved by using topological methods. The second boundary condition is $x(0)=0$ or $x^{\prime}(0)=0$.
\end{abstract}

\section{Introduction}

Asymptotic boundary value problems for second order ordinary differential equations appear when we look for radial solutions of nonlinear elliptic equations in unbounded domains with prescribed behaviour at infinity. Especially, the Dirichlet problem on the exterior of the ball with a bounded solution was studied by R. Stańczy [12]. Here, we are seeking a solution which has a bounded derivative at infinity and satisfies the Dirichlet or Neumann conditions on the boundary of the ball. It gives the following boundary value problems:

2000 Mathematics Subject Classification. 34B15, 34B40.

Key words and phrases. Asymptotic boundary value problem, nonresonant problem, resonant problem.

The author was supported by grant MNiI No. 1 P03A 00128.

ISSN 1425-6908（C) Heldermann Verlag. 


$$
\begin{aligned}
& x^{\prime \prime}=f\left(t, x, x^{\prime}\right), \quad x(0)=0, \quad \lim _{t \rightarrow \infty} x^{\prime}(t)=0, \\
& x^{\prime \prime}=f\left(t, x, x^{\prime}\right), \quad x^{\prime}(0)=0, \quad \lim _{t \rightarrow \infty} x^{\prime}(t)=0 .
\end{aligned}
$$

The first of them is nonresonant - the problem $x^{\prime \prime}=0, \quad x(0)=0=$ $\lim _{t \rightarrow \infty} x^{\prime}(t)$ has no nontrivial solutions. Thus, we can write down an integral operator with fixed points being solutions of the problem (comp. [6]). The second problem is resonant, since the corresponding homogeneous linear problem: $x^{\prime \prime}=0, \quad x^{\prime}(0)=0=\lim _{t \rightarrow \infty} x^{\prime}(t)$ has nontrivial solutions - constant functions. Hence, there is no equivalent integral equation. The existence of a solutions must be obtained by other topological tools. Similar boundary value problems have been studied by different methods in many papers. We mention only $[1,2,3,4,5,8,9,11]$.

In [10], the asymptotic boundary condition $\lim _{t \rightarrow \infty} x^{\prime}(t)=0$ is replaced by $x \in H^{2}\left(\mathbb{R}_{+}\right)$that is close but not the same as our one. Assumption are of completely different kind.

Although the radial solutions of elliptic equations take values in $\mathbb{R}$, we will consider $x \in \mathbb{R}^{k}$. Such problems for ordinary differential equations are of an independent interest.

\section{The choice of the space}

In order to apply known topological methods, we need an appropriate Banach space. Let $\mathbb{U}$ be a Banach space

$$
\mathbb{U}=\left\{x: \mathbb{R}_{+} \rightarrow \mathbb{R}^{k} \mid x \in C^{1}([0, \infty)), \lim _{t \rightarrow \infty} x^{\prime}(t)=0\right\}
$$

with the norm

$$
\|x\|_{\mathbb{U}}=\max \left\{|x(0)|, \sup _{t \in \mathbb{R}_{+}}\left|x^{\prime}(t)\right|\right\} .
$$

The convergence of the sequence $\left(x_{n}\right)$ in the space $\mathbb{U}$ means: $\left(\left.x_{n}\right|_{K}\right)$ is uniformly convergent for any compact set $K \subset[0, \infty)$ and $\left(x_{n}^{\prime}\right)$ is uniformly convergent.

Observe that $\mathbb{U}$ is a space of functions such that:

Lemma 2.1. Let $x \in \mathbb{U}$ and $\|x\|_{\mathbb{U}}=M$, then $|x(t)| \leq M(t+1)$ for any $t \in[0, \infty)$.

Proof. Observe that

$$
\begin{aligned}
|x(t)| & =|x(t)-x(0)+x(0)| \leq|x(t)-x(0)|+|x(0)| \\
& \leq t \sup _{t \in \mathbb{R}_{+}}\left|x^{\prime}(t)\right|+|x(0)| \leq M(t+1),
\end{aligned}
$$

and the lemma follows. 
The following theorem gives a compactness criterion in $\mathbb{U}$.

Theorem 2.1. For a set $A \subset \mathbb{U}$ to be relatively compact, it is necessary and sufficient that:

(1) there exists $M>0$ that for any $x \in A$ and $t \in[0, \infty)$ we have $\left|x^{\prime}(t)\right| \leq M$ and $|x(0)| \leq M$,

(2) for each $a>0$, the family $A_{a}:=\left\{x^{\prime} \mid[0, a]: x \in A\right\}$ is equicontinuous,

(3) for any $\varepsilon>0$ there exists $S>0$ such that if $t \geq S$ then $\left|x^{\prime}(t)\right| \leq \varepsilon$.

Proof. Necessity. If $A \subset \mathbb{U}$ is relatively compact then the Ascoli-Arzelà theorem implies (1) and (2). If (3) were not satisfied, there would be $\varepsilon_{0}>0$, sequence $t_{n} \rightarrow \infty$ and function $x \in A$ such that $\left|x_{n}^{\prime}(t)\right| \geq \varepsilon_{0}$ what contradicts $\lim _{t \rightarrow \infty} x^{\prime}(t)=0$.

Sufficiency. Let us consider a sequence $\left(x_{n}\right) \subset A$. By (1) we know that $\left|x_{n}^{\prime}(t)\right| \leq M$ for $t \geq 0$. We may extract a subsequence $x_{n}^{\prime 1}$ which is uniformly convergent on $[0,1]$. Now, from $x_{n}^{\prime 1}$ we extract subsequence $x_{n}^{\prime 2}$ which is uniformly convergent on $[0,2]$. Analogously, we get a sequence $x_{n}^{\prime k}$ which is uniformly convergent on $[0, k]$. Let $y_{n}^{\prime}:=x_{n}^{\prime}{ }^{n}$. This sequence is almost uniformly convergent.

By (1) we also have that $\left|y_{n}(0)\right| \leq M$. So we can extract a convergent subsequence $y_{n_{l}}(0)$. Moreover, by (3), we get

$$
\left|y_{n_{l}}^{\prime}(t)-y_{n_{m}}^{\prime}(t)\right| \leq\left|y_{n_{l}}^{\prime}(t)\right|+\left|y_{n_{m}}^{\prime}(t)\right| \leq 2 \varepsilon
$$

for $t \geq S$. Hence the sequence $y_{n_{l}}^{\prime}(t)$ is uniformly convergent for $t \geq 0$. Since $y_{n_{l}}(0)$ is convergent and $y_{n_{l}}^{\prime}$ is uniformly convergent, so we get that $\left(\left.y_{n_{l}}\right|_{K}\right)$ is uniformly convergent for any compact set $K \subset[0, \infty)$. Then the sequence $\left(x_{n}\right) \subset A$ has a convergent subsequence. Hence $A$ is relatively compact.

\section{The nonresonant problem}

Let us consider an asymptotic BVP

$$
x^{\prime \prime}=f\left(t, x, x^{\prime}\right), \quad x(0)=0, \quad \lim _{t \rightarrow \infty} x^{\prime}(t)=0,
$$

where $f: \mathbb{R}_{+} \times \mathbb{R}^{k} \times \mathbb{R}^{k} \rightarrow \mathbb{R}^{k}$ is continuous function.

The problem is nonresonant, i.e. for $f=0$, there is no nontrivial solutions, it follows that the Green function exists and (3.1) is equivalent to the integral equations

$$
x(t):=-\int_{0}^{\infty} \min (t, s) f\left(s, x(s), x^{\prime}(s)\right) d s .
$$

We will show that problem (3.1) has at least one solution. 
We will need the following assumption:

(i) $|f(t, x, y)| \leq a(t)|x|+b(t)|y|+c(t)$, where $\int_{0}^{\infty}(s+1) a(s) d s<\infty$, $\int_{0}^{\infty} b(s) d s<\infty, \int_{0}^{\infty} c(s) d s<\infty$.

Now, we consider the nonlinear integral operator $T: \mathbb{U} \rightarrow \mathbb{U}$ given by

$$
T x(t):=-\int_{0}^{\infty} \min (t, s) f\left(s, x(s), x^{\prime}(s)\right) d s .
$$

We will show that under assumptions $(i)$ operator $T$ is well defined and continuous. Let

$$
\int_{0}^{\infty}(s+1) a(s) d s=M_{1}, \quad \int_{0}^{\infty} b(s) d s=M_{2}, \quad \int_{0}^{\infty} c(s) d s=M_{3} .
$$

Since $x \in \mathbb{U}$, thus by Lemma 2.1 there exists $M>0$ such that $|x(t)| \leq$ $M(t+1)$. Observe that

$$
\begin{aligned}
& \int_{t}^{\infty}\left|f\left(s, x(s), x^{\prime}(s)\right)\right| d s \leq \int_{0}^{\infty}\left|f\left(s, x(s), x^{\prime}(s)\right)\right| d s \\
& \leq \int_{0}^{\infty} a(s)|x(s)| d s+\int_{0}^{\infty} b(s)\left|x^{\prime}(s)\right| d s+\int_{0}^{\infty} c(s) d s \\
& \leq M \int_{0}^{\infty}(s+1) a(s) d s++M \int_{0}^{\infty} b(s) d s+\int_{0}^{\infty} c(s) d s \\
& \leq M\left(M_{1}+M_{2}\right)+M_{3}<\infty
\end{aligned}
$$

under assumption $(i)$ and (3.3). We have

$$
(T x)(t)=-\int_{0}^{t} s f\left(s, x(s), x^{\prime}(s)\right) d s-t \int_{t}^{\infty} f\left(s, x(s), x^{\prime}(s)\right) d s
$$

and

$$
(T x)^{\prime}(t)=-\int_{t}^{\infty} f\left(s, x(s), x^{\prime}(s)\right) d s .
$$

Observe that $T x$ and $(T x)^{\prime}$ are continuous, which is clear from (3.5), (3.6) and (3.4). Moreover

$$
\lim _{t \rightarrow \infty}(T x)^{\prime}(t)=\lim _{t \rightarrow \infty}\left(-\int_{t}^{\infty} f\left(s, x(s), x^{\prime}(s)\right) d s\right)=0 .
$$

Hence $T: \mathbb{U} \rightarrow \mathbb{U}$.

Now, observe that operator $T$ is continuous. Let $x_{n} \subset \mathbb{U}$ and let $\left(x_{n}\right) \rightarrow x$. Since $T x(0)=0$ for any $x \in \mathbb{U}$, we have

$$
\begin{aligned}
\left\|T x_{n}-T x\right\|_{\mathbb{U}} & =\sup _{t \in \mathbb{R}_{+}}\left|\left(T x_{n}\right)^{\prime}(t)-(T x)^{\prime}(t)\right| \\
& \leq \int_{0}^{\infty}\left|f\left(s, x_{n}(s), x_{n}^{\prime}(s)\right)-f\left(s, x(s), x^{\prime}(s)\right)\right| d s .
\end{aligned}
$$


Hence we get that operator $T$ is continuous, which is clear from (3.4) and the Lebesgue dominated convergence theorem.

Now, we prove that operator $T$ is completely continuous.

Lemma 3.1. Under assumption $(i)$ operator $T$ is completely continuous.

Proof. We shall show that the image of $B:=\left\{x \in \mathbb{U} \mid\|x\|_{\mathbb{U}} \leq M\right\}$ under $T$ is relatively compact.

First, we prove condition (1) of Theorem 2.1. From (3.4) we know that there exists $L=M\left(M_{1}+M_{2}+M_{3}\right)+M_{4}$ such that for any $x \in B$ and $t \in[0, \infty)$ we have $\left|(T x)^{\prime}(t)\right| \leq L$. We thus get $(1)$.

Now, we prove condition (2). We show that for any $\varepsilon>0$ and every $t_{0} \in[0, S]$ there exists $\delta>0$ such that if $\left|t-t_{0}\right|<\delta$ then $\left|(T x)^{\prime}(t)-(T x)^{\prime}\left(t_{0}\right)\right|<\varepsilon$. By assumption $(i)$ we get that for every $\varepsilon>0$ there exists $\delta_{1}>0$ such that, if $\left|t-t_{0}\right|<\delta_{1}$ then

$$
\int_{\min \left\{t_{0}, t\right\}}^{\max \left\{t_{0}, t\right\}}(s+1) a(s) d s<\frac{\varepsilon}{3 M},
$$

$\delta_{2}>0$ such that, if $\left|t-t_{0}\right|<\delta_{2}$ then

$$
\int_{\min \left\{t_{0}, t\right\}}^{\max \left\{t_{0}, t\right\}} b(s) d s<\frac{\varepsilon}{3 M},
$$

and $\delta_{3}>0$ such that, if $\left|t-t_{0}\right|<\delta_{3}$ then

$$
\int_{\min \left\{t_{0}, t\right\}}^{\max \left\{t_{0}, t\right\}} c(s) d s<\frac{\varepsilon}{3} .
$$

Let $\delta=\min \left\{\delta_{1}, \delta_{2}, \delta_{3}\right\}$. Hence, from Lemma 2.1, we obtain

$$
\begin{aligned}
& \left|(T x)^{\prime}(t)-(T x)^{\prime}\left(t_{0}\right)\right|=\left|\int_{t_{0}}^{t} f\left(s, x(s), x^{\prime}(s)\right) d s\right| \\
& \leq \int_{\min \left\{t_{0}, t\right\}}^{\max \left\{t_{0}, t\right\}}\left|f\left(s, x(s), x^{\prime}(s)\right)\right| d s \\
& \leq \int_{\min \left\{t_{0}, t\right\}}^{\max \left\{t_{0}, t\right\}} a(s)|x(s)| d s+\int_{\min \left\{t_{0}, t\right\}}^{\max \left\{t_{0}, t\right\}} b(s)\left|x^{\prime}(s)\right| d s+\int_{\min \left\{t_{0}, t\right\}}^{\max \left\{t_{0}, t\right\}} c(s) d s \\
& \leq M \int_{\min \left\{t_{0}, t\right\}}^{\max \left\{t_{0}, t\right\}}(s+1) a(s) d s+M \int_{\min \left\{t_{0}, t\right\}}^{\max \left\{t_{0}, t\right\}} b(s) d s+\int_{\min \left\{t_{0}, t\right\}}^{\max \left\{t_{0}, t\right\}} c(s) d s \\
& <M \frac{\varepsilon}{3 M}+M \frac{\varepsilon}{3 M}+\frac{\varepsilon}{3}=\varepsilon .
\end{aligned}
$$


It remains to prove condition (3). By assumption $(i)$ for every $\varepsilon>0$ there exists $t_{1}, t_{2}, t_{3}$ large enough so that

$$
\int_{t_{1}}^{\infty}(s+1) a(s) d s<\frac{\varepsilon}{3 M}, \quad \int_{t_{2}}^{\infty} b(s) d s<\frac{\varepsilon}{3 M}, \quad \int_{t_{3}}^{\infty} c(s) d s<\frac{\varepsilon}{3} .
$$

Let $S=\max \left\{t_{1}, t_{2}, t_{3}\right\}$. From Lemma 2.1 and (3.7) for $t \geq S$ we get

$$
\begin{aligned}
& \left|(T x)^{\prime}(t)\right|=\left|-\int_{t}^{\infty} f\left(s, x(s), x^{\prime}(s)\right) d s\right| \\
& \leq M \int_{S}^{\infty}(s+1) a(s) d s+M \int_{S}^{\infty} b(s) d s+\int_{S}^{\infty} c(s) d s \\
& <M \frac{\varepsilon}{3 M}+M \frac{\varepsilon}{3 M}+\frac{\varepsilon}{3}=\varepsilon,
\end{aligned}
$$

and the proof is complete.

We will need an additional assumption:

(ii) there exists $M>0$ such that $(y \mid f(t, x, y))>0$ for $t \geq 0, x \in \mathbb{R}^{k}$ and $|y| \geq M$.

Theorem 3.1. Let assumptions $(i)-(i i)$ hold. Then problem (3.1) has at least one solution.

Proof. Consider the continuous family of BVPs:

$$
x^{\prime \prime}=\lambda f\left(t, x, x^{\prime}\right), \quad x(0)=0, \quad \lim _{t \rightarrow \infty} x^{\prime}(t)=0,
$$

depending on a parameter $\lambda \in[0,1]$. Then problem (3.8) is equivalent to an integral equation

$$
\varphi(t):=-\lambda \int_{0}^{\infty} \min (t, s) f\left(s, \varphi(s), \varphi^{\prime}(s)\right) d s .
$$

By Lemma 3.1 we get that operator $\lambda T$ is completely continuous. Let us consider homotopy $H:[0,1] \times \mathbb{U} \rightarrow \mathbb{U}$ given by

$$
H(\lambda, \varphi)=\varphi-\lambda T \varphi
$$

in $\Omega=B(0, M)$.

If $H(\lambda, \varphi)=0$ for $\lambda=0$ and $\varphi \in \partial \Omega$, then $\operatorname{BVP}(3.8)$ has only a trivial solution, which does not lay on the boundary of $\Omega$, a contradiction.

Assume that $H(\lambda, \varphi)=0$ for $\lambda \in(0,1]$ and $\varphi \in \partial \Omega$. Let us consider $\psi(t)=\left|\varphi^{\prime}(t)\right|^{2}$. Observe that $\lim _{t \rightarrow \infty} \psi(t)=0$. Hence $\psi$ has maximum $M^{2}$ for certain $t_{0} \in \mathbb{R}_{+}$. If $t_{0}=0$, then from assumption $(i i)$, since $\left|\varphi^{\prime}\left(t_{0}\right)\right|=M$, we get

$$
0 \geq \psi^{\prime}\left(t_{0}\right)=2 \lambda\left(\varphi^{\prime}\left(t_{0}\right)\right) \mid f\left(t_{0}, \varphi\left(t_{0}\right), \varphi^{\prime}\left(t_{0}\right)\right)>0
$$


a contradiction. If $t_{0}>0$, then

$$
0=\psi^{\prime}\left(t_{0}\right)=2 \lambda\left(\varphi^{\prime}\left(t_{0}\right)\right) \mid f\left(t_{0}, \varphi\left(t_{0}\right), \varphi^{\prime}\left(t_{0}\right)\right)>0
$$

a contradiction. Hence homotopy $H$ does not vanish on the boundary of $\Omega$ for $\lambda>0$. Finally $H(\lambda, \varphi) \neq 0$ for $\lambda \in[0,1]$ and $\varphi \in \partial \Omega$. Consequently,

$$
\begin{aligned}
\operatorname{deg}_{L S}(I-T, \Omega) & =\operatorname{deg}_{L S}(H(1, \cdot), \Omega)=\operatorname{deg}_{L S}(H(0, \cdot), \Omega)=\operatorname{deg}_{L S}(I, \Omega) \\
& =1 \neq 0 .
\end{aligned}
$$

Hence $T$ has a fixed point in $\Omega$. We obtain that problem (3.1) has at least one solution.

\section{The resonant problem}

Let us consider an asymptotic BVP

$$
x^{\prime \prime}=f\left(t, x, x^{\prime}\right), \quad x^{\prime}(0)=0, \quad \lim _{t \rightarrow \infty} x^{\prime}(t)=0
$$

where $f: \mathbb{R}_{+} \times \mathbb{R}^{k} \times \mathbb{R}^{k} \rightarrow \mathbb{R}^{k}$ is continuous. Since constant functions are solutions for $f=0$, the problem is resonant.

We will need the following assumptions:

(iii) $f(t, x, y)$ is Lipschitz continuous with respect to $x$ and $y$;

(iv) $\quad|f(t, x, y)| \leq b(t)|y|+c(t)$, where $\int_{0}^{\infty} b(s) d s<\infty, \int_{0}^{\infty} c(s) d s<\infty$;

(v) there exists $M>0$ such that $x_{i} f_{i}(t, x, y)>0$ for $t \geq 0, y \in \mathbb{R}^{k}$, $x \in \mathbb{R}^{k}$ and $\left|x_{i}\right| \geq M, i=1, \ldots, k$.

Now, we consider problem

$$
y^{\prime}=f\left(t, c+\int_{0}^{t} y, y\right), \quad y(0)=0,
$$

for fixed $c \in \mathbb{R}^{k}$. Observe that (4.2) is equivalent to an initial value problem

$$
x^{\prime \prime}=f\left(t, x, x^{\prime}\right), \quad x(0)=c, \quad x^{\prime}(0)=0 .
$$

Since $f$ is continuous than by assumption ( $i i i)$ and the Local Existence and Uniqueness Theorem we get that problem (4.3) has a unique local solution.

We can write down (4.2) as

$$
y_{c}(t)=\int_{0}^{t} f\left(s, c+\int_{0}^{s} y(u) d u, y(s)\right) d s .
$$

By $(i v)$ and (3.3) we get

$$
\left|y_{c}(t)\right| \leq \int_{0}^{t} b(s)|y(s)|+c(s) d s \leq M_{3}+\int_{0}^{t} b(s)|y(s)| d s .
$$


Now, due to Gronwall's Lemma, we have

$$
\left|y_{c}(t)\right| \leq M_{3} \exp \int_{0}^{t} b(s) d s
$$

Hence, by the theorem on a priori bounds [7], (4.3) has a unique global solution for $t \geq 0$. We obtain that (4.2) has a unique global solution for $t \geq 0$. Moreover, by assumption (iv) and (3.3) we have

$$
\left|y_{c}(t)\right| \leq M_{3} \exp \int_{0}^{t} b(s) d s \leq M_{3} \exp \int_{0}^{\infty} b(s) d s=M_{3} \exp M_{2}<\infty .
$$

Hence (4.2) has a unique bounded global solution for $t \geq 0$.

The function $t \mapsto f\left(t, c+\int_{0}^{t} y(u) d u, y(t)\right)$ is absolutely integrable, i.e.

$$
\forall_{\varepsilon>0} \quad \exists_{M>0} \quad\left|\int_{M}^{\infty} f\left(t, c+\int_{0}^{t} y(u) d u, y(t)\right) d t\right|<\varepsilon .
$$

In particular, there exists a limit

$$
g(c):=\lim _{t \rightarrow \infty} y_{c}(t)
$$

We conclude that $g: \mathbb{R}^{k} \rightarrow \mathbb{R}^{k}$.

Lemma 4.1. Under assumptions (iii) and (iv) $g$ is continuous.

Proof. Fix $c_{0} \in \mathbb{R}^{k}$ and $\varepsilon>0$. We choose $M>0$ large enough to get

$$
\left|\int_{M}^{\infty} f\left(t, c+\int_{0}^{t} y(u) d u, y(t)\right) d t\right| \leq L \int_{M}^{\infty} b(t) d t+\int_{M}^{\infty} c(t) d t<\frac{\varepsilon}{3} .
$$

Now, due to the continuous dependence of solution with respect to parameter, we have that there exists $\delta>0$ such that, if $\left|c-c_{0}\right|<\delta$ then $\left|y_{c}(M)-y_{c_{0}}(M)\right|<\varepsilon / 3$.

We get

$$
\begin{aligned}
\left|y_{c}(\infty)-y_{c_{0}}(\infty)\right| & \leq\left|y_{c}(\infty)-y_{c}(M)\right|+\left|y_{c}(M)-y_{c_{0}}(M)\right|+\left|y_{c_{0}}(M)-y_{c_{0}}(\infty)\right| \\
& \leq 3(\varepsilon / 3)=\varepsilon,
\end{aligned}
$$

which completes the proof.

The solution of the problem (4.1) is obtained, if one can find such $c \in \mathbb{R}^{k}$ that $g(c)=0$. To this end we need Miranda's theorem $([7])$.

Theorem 4.1. If $g: \mathbb{R}^{k} \supset[-M, M]^{k} \rightarrow \mathbb{R}^{k}$ is continuous and if:

$$
g_{i}\left(c_{1}, \ldots, c_{i-1},-M, c_{i+1}, \ldots, c_{k}\right) \leq 0
$$

and

$$
g_{i}\left(c_{1}, \ldots, c_{i-1}, M, c_{i+1}, \ldots, c_{k}\right) \geq 0
$$


for $i=1, \ldots, k$, then there exists $c \in[-M, M]^{k}$ such that $g(c)=0$.

We can now formulate our main result.

Theorem 4.2. Under assumption (iii)-(v) problem (4.1) has at least one solution.

Proof. Let $y_{c}(t)$ is the unique bounded global solution of (4.2) and $g(c):=$ $\lim _{t \rightarrow \infty} y_{c}(t)$. Observe that $x(t)=c+\int_{0}^{t} y_{c}(s) d s$ is a solution of (4.1) if there exists an $c \in \mathbb{R}^{k}$ such that $g(c)=0$.

We shall show that $g$ satisfies assumptions of Miranda's theorem. The function $g$ is continuous by Lemma 4.1 .

Let $c_{i}=M+1$. We prove that $y_{i}(t) \geq 0$ for $t \geq 0$. From (4.2) we have $y_{i}(0)=0$. Assume that for some $\mathrm{t}$ we have $y_{i}(t)<0$. Then there exists $t_{*}:=\inf \left\{t \mid y_{i}(t)<0\right\}$ such that, $y_{i}\left(t_{*}\right)=0$ and $y_{i}(t) \geq 0$ for $t<t_{*}$. Since $y_{i}(t)$ is continuous there exists $t_{1}>t_{*}$ such that

$$
\int_{t_{*}}^{t_{1}}\left|y_{i}(t)\right| d t \leq 1
$$

Hence, we get

$$
x_{i}(t)=c_{i}+\int_{t_{*}}^{t} y_{i}(s) d s M+1+\int_{t_{*}}^{t} y_{i}(s) d s \geq M \quad \text { for } t \in\left[t_{*}, t_{1}\right] .
$$

Now, by condition $(v)$ we get

$$
x_{i}(t) f_{i}(t, x(t), y(t))=x_{i}(t) y_{i}^{\prime}(t) \geq 0 .
$$

Hence $y_{i}^{\prime}(t) \geq 0$ for $t \in\left[t_{*}, t_{1}\right]$. It means that $y_{i}(t)$ is nondecreasing on $\left[t_{*}, t_{1}\right]$. Since $y_{i}\left(t_{*}\right)=0$ we get a contradiction. Hence $y_{i}(t) \geq 0$ for $t \geq 0$. In consequence

$$
g_{i}\left(c_{1}, \ldots, c_{i-1}, M+1, c_{i+1}, \ldots, c_{k}\right)=\lim _{t \rightarrow \infty} y_{i}(t) \geq 0
$$

for $i=1, \ldots, k$. To prove condition

$$
g_{i}\left(c_{1}, \ldots, c_{i-1},-M-1, c_{i+1}, \ldots, c_{k}\right) \leq 0
$$

we can proceed analogously. Hence there exists an $c \in[-M-1, M+1]^{k}$ such that, $g(c)=0$. This completes the proof.

Remark. One can replace boundary condition $x(0)=0$ in $(3.1)$ and $x^{\prime}(0)=$ 0 in (4.1) by nonhomogeneous ones. Arguments for proving the existence of a solution under the same assumptions are standard. 


\section{References}

[1] Andres, J., Gabor, G., Górniewicz, L., Boundary value problems on infinite intervals, Trans. Amer. Math. Soc. 351 (1999), 4861-4903.

[2] Constantin, A., On an infinite interval boundary value problem Ann. Mat. Pura Appl. (4) 176 (1999), 379-394.

[3] Bebernes, J. W., Jackson, L. K., Infinite interval boundary value problems for $y^{\prime \prime}=$ $f(x, y)$, Duke Math. J. 34 (1967), 39-47.

[4] Furi, M., Pera, M. P., A continuation method on locally convex spaces and applications to ordinary differential equations on noncompact intervals, Ann. Polon. Math. 47 (1987), 331-346.

[5] Karpińska, W., On bounded solutions of nonlinear differential equations at resonanse Nonlinear Anal. 51 (2002), 723-733.

[6] Mawhin, J., Topological degree methods in nonlinear boundary value problems, CBMS Regional Conference Series in Mathematics 40, Amer. Math. Soc., Providence, R.I., 1979.

[7] Piccinnini, L. C., Stampacchia, G., Vidossich, G., Ordinary Differental Equations in $\mathbb{R}^{n}$, Appl. Math. Sci. 39, Springer-Verlag, New York-Heidelberg-Berlin, 1984.

[8] Przeradzki, B., On a two-point boundary value problem for differential equations on the half-line, Ann. Polon. Math. 50 (1989), 53-61.

[9] Przeradzki, B., Nonlinear equations with non-Fredholm linear part, Nonlinear Anal. 47 (2001), 4917-4923.

[10] Rabier, P. J., Stuart, C. A., A Sobolev space approach to boundary value problems on the half-line, Commun. Contemp. Math. 7(1) (2005), 1-36.

[11] O'Regan, D., Solvability of some singular boundary value problems on the semiinfinite intevral, Canad. J. Math. 48 (1) (1996), 143-158.

[12] Stańczy, R., Bounded solutions for a nonlinear elliptic equations in unbounded domains, J. Appl. Anal. 6(1) (2000), 129-138.

KATARZYNA SZYMAŃSKA

Centrum NaucZania Matematyki i Fizyki

POLITECHNIKA EÓDZKA

Al. Politechniki 11

90-924 ŁÓDŹ, POLAND

E-MAIL: GRAMPA@ZB.NET.LODZ.PL 